\title{
KNOWLEDGE LIMITS IN COSMOLOGY
}

\author{
MENAS KAFATOS \\ Earth Observing and Space Research Program \\ Institute for Computational Sciences and Informatics \\ and Department of Physics \\ George Mason University \\ Fairfax, VA 22030
}

\begin{abstract}
In cosmology one faces the observational challenge that knowledge about distant regions of the universe is dependent on assumptions one makes about these regions which are themselves coupled to the observations. Within the framework of the Friedmann-Robertson-Walker big bang models the universe becomes opaque to its own radiation at $\mathrm{z} \approx 1,000$ and the earlier, and more distant, regions of the universe are not directly accessible through observations. Other challenges exist such as possible merging of extended distant sources and confusion of spectra from distant galaxies. One, therefore, encounters horizons in our understanding of the universe. Such horizons exist in any mode of description. To use the quantum analogy, the observer is always part of the system under study, the universe, and a description of the universe entails including the observer and observing apparatus. Since the early universe should be described in quantum terms, it follows that non-locality in the universe is not an a-priori requirement but the outcome of the observing process itself. As such, the flatness and horizon problems may not be preconditions on theoretical models.
\end{abstract}

\section{Introduction}

It was Einstein's general theory of relativity that allowed the possibility of an evolving, non-static, universe. The cosmological revolution would prove of equally great significance as the quantum revolution -- both broke away from traditional thinking. With the theoretical framework of general relativity already in place, the Belgian cosmologist Abbe Lemaitre and the Russian mathematician Alexander Friedmann postulated in the early 20's a dynamic, expending and evolving universe. The Friedmann models obeyed the cosmological principle, which states that the universe is isotropic -- the same in all directions -- and homogeneous -- of equal density, on the average, everywhere. It then followed that as the universe expands, the average density of matter would decrease. To accommodate the obvious observational picture of Hubble's expanding universe with a framework of an eternal universe, Herman Bondi, Thomas Gold and Fred Hoyle 
proposed in the late 40's the steady state theory, describing a universe which although expanding would obey the perfect cosmological principle: The universe would appear the same to all observers at all times. What Bondi, Gold and Hoyle were attempting to avoid was the question of the origins of the universe: If the universe is expanding, Lemaitre and the other cosmologists of the early 20's reasoned, it must have been much denser and hotter in the distant past, it must have started from a primordial singularity. Bondi, Gold and Hoyle avoided the problem of a primordial dense universe at the expense of a cherished principle of physics, the principle of conservation of mass and energy. The steady state model demanded the creation of matter from nothing, new matter had to be created to fill the voids of expanding space.

In the early 50's, cosmologist George Gamow extended Lemaitre's and Friedmann's original ideas by incorporating nuclear physics. Cosmologists could now use nuclear physics to speculate what might have happened in the early lifetime of the universe when it was very hot and prone to nuclear interactions.

Soon observational astronomy armed with the new branch of millimeter and radio astronomy and sophisticated optical spectroscopy provided strong evidence in favor of the big band model, through existence of the $3^{\circ} \mathrm{K}$ black body radiation and the existence of quasars. In the big bang picture, the microwave background is the relic radiation from the initial big bang. The steady state theory could not as easily account for the background.The microwave radiation can be easily accounted for by the big bang theory and, it appeared no extra assumptions were needed.

The second cosmological observation that challenges steady state is the existence of the quasars (Berry 1976). These objects appear to be very distant, some of them receding away from us at speeds exceeding $80 \%$ of c. In the general "standard" scenario, at the distance of a quasar, only the brilliant star-like nucleus can be seen. These bright nuclei of galaxies were very brilliant in the past compared to the present era, indicating that sources evolved as time went on. If the interpretation is correct, quasars would violate the perfect cosmological principle because the universe would not look the same at all times. Recently, it has become obvious that the big bang theory itself faces theoretical challenges not appreciated before. Yet, the vast majority of astronomers, cosmologists and particle physicists still adhere to the big bang theory, which has become the gospel of cosmological theory.

One should, however, be cautious in embracing a single vision of the universe. Even though the standard theory has had substantial successes, the mystery of quasars has still not been completely unravelled. Moreover, modern challenges have emerged, such as the remarkable smoothness of the microwave background, the increasing complexity of assumptions tied to cold dark matter (CDM) theory and as recent HST observations indicate, the perplexing emergence of a relatively young universe at odds with both stellar evolution and the inflationary predictions. As such, perhaps a re-examination of cosmological theories and a possible convergence and acceptance of, seemingly, opposing views may be warranted. It is my purpose here to examine the limits of both cosmological theories and observations and to provide some new approaches to cosmology. 


\section{The Early Universe and Inflation}

Recent advances in particle theory have afforded us unique opportunities to describe the conditions of the early universe. The early universe was in a quantum state and moreover, the early universe can be used as a cosmic laboratory to push physics to the ultimate frontier of quantum gravity.

Very near the original singularity, the space-time description breaks down entirely. This is followed by the so-called "inflationary era " at about $10^{-35} \mathrm{sec}$ (Guth and Steinhardt 1984). During the inflationary era, the universe underwent an extremely rapid expansion doubling in size every $10^{-35}$ of a second. By the time the universe had finished through this phase, it had expanded in size by a staggering factor of $10^{50}$ of more. Prior to inflation, the universe was in a phase of symmetry with respect to the so-called Higgs fields -- and the strong, weak and electromagnetic interactions were unified. In that situation the Higgs fields, members of a special set of quantum fields postulated in Grand Unified Theories (GUT) to account for spontaneous symmetry breaking, were all zero. At a temperature of about $10^{27} \mathrm{~K}$, the universe underwent a phase transition from the false vacuum where all the Higgs fields were zero to a less energetic phase, the true vacuum of quantum theory. In the true vacuum state (Barrow 1988), the Higgs fields acquired non-zero values and the GUT symmetry broke down. During the inflationary era, the non-zero values of the Higgs fields broke down the GUT symmetry: The strong force separated from the electroweak force.

The false vacuum of GUT has many peculiar properties, the most peculiar being perhaps the form of its negative pressure. In those conditions, general relativity predicts that gravity rather than pulling together would be pushing away. The vast energies locked up in the negative pressure of the false vacuum were released and all matter formed.

The inflationary model was originally proposed not because of a compelling theoretical reason but in order to address some observational problems faced by standard big bang. For example, the horizon of the universe, within which parts of the expanding primordial matter were in contact among themselves, expanded becoming much larger than the radius of the observable universe. In the big bang cosmology without inflation the horizon is always less than the radius of the observable universe. The "true" universe would then be expected to be much larger than the universe we can see observationally. This fact is critical to resolve one of the observational problems of the standard big bang without inflation namely, the horizon problem.

After inflation released the vast amounts of energy that would later on coalesce to form all the observable objects in the universe, the expansion proceeded according to the original version of the big bang theory. Between $10^{30} \mathrm{sec}$ and $10^{-6} \mathrm{sec}$ the universe was filled with a primordial soup of quarks and leptons. After about $10^{-6} \mathrm{sec}$, the quarks, combined together to form the nucleons. In the next phase, between $1 \mathrm{sec}$ and 3 minutes, protons and neutrons underwent nuclear reactions forming nuclei of helium and its isotopes.

Until about $\sim 100,000$ years after the big bang, photons and matter were coupled together. The $2.735^{\circ} \mathrm{K}$ black body photons were also emitted at this time but provided no opportunity following this era to probe the earlier periods where fundamentally 
important physical processes were taking place. It follows that events in these earlier periods cannot be verified by means of the main tool of observational astronomy -- quanta of light.

\section{Observational Limits in Cosmology}

The big bang theory of the universe could not account for a number of features revealed by observational cosmology such as the so-called flatness problem. Quantitatively this is expressed as $\Omega=\rho / \rho_{\text {crit }}$ and the critical density can be expressed as (Barrow 1988) $\rho_{\text {crit }}=5 \times 10^{-30}\left(\mathrm{H}_{\mathrm{o}} / 50 \mathrm{~km} / \mathrm{sec} / \mathrm{Mpc}\right)^{2} \mathrm{gr} \mathrm{cm}^{-3}$

where $\mathrm{H}_{\mathrm{o}}$ is the Hubble constant. It is obvious that the rate of expansion cannot increase and infinitum. There would be a point when the rate of expansion as seen from the earth would reach the speed of light. This would constitute the horizon of the universe. For $\mathrm{H}_{\mathrm{o}}=50 \mathrm{~km} / \mathrm{sec} / \mathrm{Mpc}$ that horizon lies $\sim 20$ billion light years away from the earth. The precise value of $\mathrm{H}_{\mathrm{o}}$ remains the most fundamental challenge of observational cosmology (see present volume). Were $\Omega$ turn out to be precisely equal to unity the geometry of the universe would be exactly flat. Inflation nicely accounts for the apparent flatness since no matter what the original curvature was, the inflationary era washed it out into perfect flatness.

Current observations cannot unequivocally distinguish the type of the universe we line in. Values of $\Omega$ for luminous matter are in the range $\leq 0.1$ to even as large as 2 although most observers favor values $\mathbf{3} 0.1$. If the only type of matter existing in the universe is luminous matter, this result would favor an open universe. Even though present observations only indicated an approximate range of the mean density of the universe, this range is so close to the value of the critical density required for a flat geometry that many astronomers assume as a working hypothesis that the universe is exactly flat, hence the need for unseen forms of matter such as cold dark matter (CDM).

The second problem facing big bang cosmology has to do with the uniformity of the $3^{\circ} \mathrm{K}\left(2.735^{\circ} \mathrm{K}\right.$ to be more exact) black body radiation (see discussion in present volume). The microwave radiation has the same temperature to within one part in $\geq 10,000$ in every direction of the sky. In the hot big bang, though, opposite parts in the sky at the time that the microwave background formed $10^{5}$ years from the beginning, were separated by distances of $10^{7}$ light years (Schramm 1983). Given the near identity of temperatures from all parts of the sky and presuming that classical causality holds, one would conclude that opposite parts of the sky had to be in casual contact. Relativity, though, states that no signal can travel faster than light, and opposite parts of the sky were spacelike in their separation. This is known as the horizon problem, and as with the flatness problem, it represents incredibly fine tuning in the conditions prevailing in the early universe.

Big bang models of the universe assume that the universe is isotropic and homogeneous. This may be difficult to achieve given the large number of possibilities in the initial conditions. In fact, quantitative calculations show that slight anisotropies would not die away but on the contrary would get amplified. This is known as the isotropy 
problem. The problem becomes even more severe in the steady state scenario which requires that whatever fluctuations in isotropy exist should be present at all levels. Whether the universe is isotropic and homogeneous is an observational question. The microwave radiation is highly isotropic. The universe is also presumed to be expanding the same way in all directions, i.e. to be isotropic in matter as it is in background radiation. And the distribution of matter is presumed to reach homogeneity beyond the largest structures seen, the superclusters, i.e. beyond hundreds of Mpc.

Recent observations challenge both these pillars of traditional cosmological thinking: The universe may not be homogeneous, larger and larger structures have been found. Galaxies seemingly cluster themselves to increasing hierarchies of clusters, superclusters and maybe even super-superclusters. Often these structures assume the form of filaments on the surface of very large bubbles with large "voids" in between (Geller and Huchra 1989). The universe may also not be isotropic, galaxies have been found which do not follow the Hubble flow (Dressler et al. 1987).

One would, therefore, conclude that the universe requires incredibly fine tuning at the beginning, i.e. the universe represents a very unlikely "accident". Paul A.M. Dirac (1937) first noticed in the 30's that certain ratios involving fundamental constants of nature and physical parameters obey simple numerical relations. Some of these coincidences yield very large numbers which are not random as one might have expected. Dirac believed these could not be coincidences and formulated his large number hypothesis. Simply put, he reasoned that since as the universe expands its radius changes in value; in order for various ratios to be equal today, a physical quantity such as Newton's gravitational constant must change in time. Attempts to verify Dirac's hypothesis have failed.

Today some physicists, primarily Dicke, Carter, Barrow and Tipler favor another approach. They have postulated that our existence as observers requires a fine tuning and that the seemingly unrelated radios of different quantities point to our existence as necessitating the kind of universe we live in. Put differently, the universe is unique because it contains conscious observers. This is known as the anthropic principle (Barrow \& Tipler 1986).

\section{Horizons of Knowledge in Cosmological Theory}

As we study the observations pertaining to the early universe, we encounter a number of observational horizons of knowledge (Kafatos, 1989). These observational horizons are tied to the quantum nature of photons. For example, to obtain the distance of a faint galaxy requires that we obtain its spectrum, which in turn requires that we disperse the light. This requires isolating the light from the galaxy by means of a narrow slit. At larger distances, when few photons are involved, one cannot disperse the light without limit. Attempting to obtain more photons by decreasing the dispersion would, on the other hand, cause an observational confusion as light from neighboring galaxies in that part of the sky would also fall onto the spectrograph. There is then a complementary inverse relationship between dispersion and brightness which does not permit accurate 
spectra of faint, very distant galaxies to be obtained (practically, this limit does not apply to the current telescopes, including HST).

When we study the predictions of various competing models of the universe we find that the observational horizons complicate the theoretical picture. Moreover (Kafatos 1989), theoretical models present us with their own limitations, what one may call theoretical horizons of knowledge.

For example, big bang cosmology itself imposes a fundamental limit on the observability of the early universe. Direct observations of the early universe based on photons provide information only after a timescale of $\sim 100,000$ years from the beginning. One hundred thousand years after the beginning corresponds to $z$ of $\sim 1000$, i.e. when the universe was only $0.1 \%$ of $1 \%$ of its present age. On the other hand, the most distant quasars are seen at a redshift of $\geq 4$ and emitted their light received by us today when the universe was about $10 \%$ of its present age. Radiation can in principle tell us much more about the early universe than matter. However, the opaqueness of the universe prior to $z \sim 1000$ simple does not allow us to confirm or deny big-bang cosmology based solely on photons. At $z \sim 1000$ we encounter the first theoretical horizon of knowledge about our universe and as long as we are constrained to observe photons, that horizon is impregnable (Kafatos 1989).

It is unlikely that any other means will provide as clear-cut evidence as light about the universe we live in. Even though neutrino astronomy is an exciting new branch of observational astronomy, it cannot replace traditional astronomy and its main tool, light. In principle, primordial neutrinos emitted a few seconds after the beginning of the universe or at a redshift $\mathrm{z} \sim 10^{9}$ present, therefore, the ultimate horizon from which we can access direct information about he universe (Kafatos 1989).

Relying on ordinary matter can yield a lot of detailed information about the hypothesis of element formation in the early universe (Schramm 1983). The actual situation is in reality difficult, since uncertainties in the abundances of the primordial elements deuterium, helium and ${ }^{7} \mathrm{Li}$ are large and the details of big bang models are least sensitive to the abundance of helium, by far the most abundant of primordial elements formed from hydrogen. Present results, if no CDM exists, imply a mean density of baryons $\approx 3 \times 10^{-31} \mathrm{gr} / \mathrm{cm}^{3}, 2$ orders of magnitude less than the critical density, implying an open universe. The corresponding horizon of knowledge associated with nucleosynthesis is $\mathrm{z} \sim 10^{8}$.

Of all the horizons of knowledge at $\mathrm{z} \sim 10^{9}, 10^{8}$, and 1000 , it is the last that is likely to remain the only one we can explore for the foreseeable future. The neutrino horizon at $10^{9}$ is not going to be qualitatively different from the photon horizon because both primordial neutrinos and photons follow a black body radiation law. Whatever problems of interpretation we are facing today with regards to the background photons will not go away with neutrinos, except that the problem of detection and interpretation will only be much worse. And the element horizon going back to the first three minutes will not yield much better information either due to the complexity of nuclear reactions applicable at that time or the uncertainties of whether these elements represent truly primordial matter.

Considerations from classical cosmological theory can shed more light as to the type of the universe we live in, by studying the Hubble Diagram for distant galaxies. 
Redshifts in the range $5 \sim 10$ would be particularly important to study since astronomers suspect that at these redshift galaxies began to form. It is conceivable though that distant galaxy images would merge at large, $\geq 30$, redshifts, and it would be virtually impossible to obtain accurate spectra to study the geometry of the universe. The curvature of the universe causes the image to decrease for redshifts greater than the minimum value which occurs for $z \sim 1$ (Narlikar 1983). Although presently inaccessible, this "galaxy image" theoretical limit may one day be reachable, if indeed there were any galaxies out to $\mathrm{z} \geq 30$.

These horizons which occur inherently in the particular theoretical picture used become worst when the quantum nature of light is considered: Spectra of different sources in the sky would themselves be blended together as one looks at fainter and fainter sources. Eventually, the background from different faint galaxies would dominate the spectrum from a single distant galaxy and reliable spectra could not be obtained. It is for these reasons, that we encounter cosmological horizons of knowledge which prevent us from ultimately deciding unequivocally how these tests confirm or reject particular theoretical models (Kafatos 1989). This is precisely the case, where Bohr insisted, complementarity acquires great importance. One would then view the various cosmological models not as rival theories of which one day only one will emerge as the theory of the universe, but as competing complementary constructs. Coupled with the fact that the early universe should be described in quantum terms, one would conclude that these emergent complementary models and the implied underlying wholeness are not an a-priori philosophical preference but the very outcome of the observing process for the early universe.

As such, the flatness, horizon and isotropy problems may be tied to the observing process itself rather than as preconditions for theory. As we look at more and more distant galaxies, the universe may be appearing as flat not because of inflation but rather, because such a universe would naturally emerge as the boundary between complementary constructs: The open versus closed universe constructs. One should perhaps view big bang and steady state models as complementary constructs as well. Although clearly favored at present, big bang theory may reveal further weaknesses as the observational limits are extended.

One final point that $\mathrm{I}$ would like to raise is the intriguing possibility that quantum-like non-locality prevailed even after the early quantum gravity and quantum inflationary any era. This would provide an alternative path to the correlations implied by the COBE results. As such, Bell-type quantum correlations may be frozen in (Kafatos 1989). We may indeed have to take our own views of the quantum nature of the early universe much more seriously. In that case, the implications not just for cosmology but for the general epistemology of science would be staggering (Kafatos and Nadeau 1990). 


\section{References}

Barrow, J.D. (1988), Q.J1. R. Astr. Soc. 29, 101-117.

Barrow, J. D. and Tipler, F.J. 1986, The Anthropic Cosmological Principle, Oxford Univ. Press, Oxford.

Berry, M. (1976), Principles of Cosmology and Gravitation, Cambridge Univ. Press, Cambridge.

Dirac, P.A.M. (1937), Nature 139, 323.

Dressler, A. et al. (1987), Ap. J. 236, 531.

Geller, M. J. and Huchra, J. (1989), Science 246, 897.

Guth, A. and Steinhardt, P.J. (1984), Scientific American, June 1984, 116-128.

Kafatos, M. (1989) in Bell's Theorem Quantum Theory and Conceptions of the Universe, ed. M-Kafatos, 195, Kluwer Academic.

Kafatos, M. and Nadeau R. (1990), The Conscious Universe: Part and Whole in Modern Physical Theory.

Narlikar, J. (1983), Introduction to Cosmology, Jones and Bartlett, New York.

Rowan-Robinson, M. (1985), The Cosmological Distance Ladder, W.H. Freeman and Co., New York.

Schramm, D.N. (1983), Physics Today, April 1983, 27-33. 